\title{
Axial Turbine Performance Estimation During Dynamic Operations
}

\author{
Razvan Nicoara $^{1}$ (D) Valeriu Vilag $^{1} \cdot$ Jeni Vilag $^{1} \cdot$ Zoltan Kolozvary $^{2}$
}

Received: 9 January 2020 / Revised: 6 July 2020 / Accepted: 21 July 2020 / Published online: 4 August 2020

(c) The Author(s) 2020

\begin{abstract}
During service, a turbine engine undergoes frequently dynamic operations. Because of the changes in mass flow, temperature, pressure, as well as the different reaction of the components of turbine to these changes, the turbine performances may vary significantly. It is critical to understand and quantify these variations to design and integrate the turbine to the engine. This paper presents a study of the performances and the variation of the performances for an axial gas turbine across its operating envelope. Numerical simulations are used to describe the flow across the turbine and to estimate the turbine maps. Using theoretical charts for typical turbine thermal expansion, the variation of tip clearance across the working line is approximated. Because of the changes of the tip clearance, using a single geometry may not supply relevant information during real dynamic operations. In order to eliminate these additional errors in the numerical study, a number of turbines maps are recalculated for different tip clearances, respectively to engine regimes. With the resulting data, the working line is recalculated across multiple maps. The comparison between the two working lines is presented as well as the study of performance variation of the turbine across the operation envelope and the influence of tip clearance at different regimes.
\end{abstract}

Keywords Fluid dynamics $\cdot$ Turbine $\cdot$ Numerical simulations $\cdot$ Maps $\cdot$ Dynamic operations

\section{Introduction}

The flow across the gap between the rotor blade and the stationary turbine case, known as blade tip-leakage flows, constitute a major source of inefficiency in the turbine, accounting for up to $1 / 3$ of the overall losses in a turbine stage [1]. The working fluid passes through this gap, thus the potential energy existing in this part of the fluid it is not extracted. Because of the rotational speed and the relative movement between the rotor blade and the casing, in the tip clearance area, a vortex forms and it is directed in the blade passage by the pressure difference between the pressure and the suction side of the rotor blade. Here, the vortex interacts with passage vortices to form a complex flow pattern. In general, the efficiency decrease linear with the increase of the clearance gap size, although the line does not pass through the origin,

Razvan Nicoara

razvan.nicoara@comoti.ro

1 Department of Aviation and Industrial Gas Turbines, Romanian Research and Development Institute for Gas Turbine, COMOTI, 061126 Bucharest, Romania

2 SC Plasmaterm SA, 540390 Târgu Mureș, Romania and the relation is presumably nonlinear at very small gaps (less than $1 \%$ chord) [2].

In the designing phase a small clearance is desirable to minimize the flow across this gap, thus minimize the losses, but a minimum gap must be provided to avoid the potential rub between the two elements. Because of the centrifugal forces on the rotor and the different thermal inertia of these elements, the tip clearance gap is not constant across the engine operating envelope, moreover, the variation is not linear. Hany Moustapha et al. [3] described the variation of tip clearance through a typical engine cycle and stated that during acceleration, due to a rapidly growing in rotational speed and centrifugal forces, rub between rotor blade tip and turbine casing is possible. During the deceleration another rub can occur if there is a full power demand at the end of the deceleration phase. Figure 1 describes the variation of the clearance tip during the acceleration and deceleration process.

In the context of developing a $400 \mathrm{~N}$ microjet engine, an axial turbine is designed to drive a centrifugal compressor. A particular problem occurs when the fuel mass flow is calculated for the dynamic regimes, especially for the lower engine speeds. Since the turbine theoretical map is calculated with the same clearance gap for all the operational 


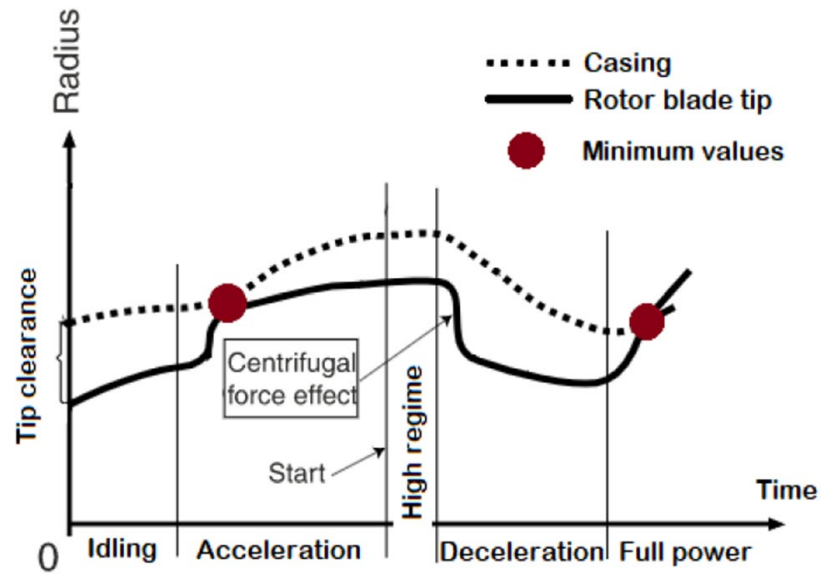

Fig. 1 Variation of tip clearance through a typical engine cycle (constructed using [3])

power settings, during the dynamic regimes the clearance gap changes as a result of different elements expansion (or contraction), thus at this operational conditions the turbine map information are not precise.

This fact leads, in case of acceleration, to a high turbine inlet temperature, when the fuel mass flow is too high as a result of underestimating the turbine power, or a slow acceleration when the mass flow is too small as a result of overestimating the turbine power.

The present study numerically investigated the effect of tip clearance and the tip clearance variations to turbine and engine performance. For this study, it was considered that the turbine follows the typical tip clearance variation trend presented in Fig. 1. Thus, no heat transfer analysis was conducted, the blade geometry was considered constant and the tip clearance variation was determined by the variation of the turbine case diameter.

\section{Numerical Setup}

The small size turbine consists of a single-stage axial flow with both, the vanes and the blades, designed with constant profile across the radius. The turbine geometric information is presented in Table 1 and the 3D models for the vane and the rotor are presented in Fig. 2.

The grid is structured and has approximately $1,800,000$ nodes in total, with the smallest cells close to the walls. To reduce the computational time, the numerical study is conducted on a single channel using periodic boundaries condition. At the interface between the stator part and the rotor part, the frozen rotor model is used. Figure 3 presents the grid for the vane and the blade.

At the inlet, it was imposed the absolute pressure, 472,600 $\mathrm{Pa}$, and the absolute temperature, $1173 \mathrm{~K}$. The
Table 1 Turbine information

\begin{tabular}{lll}
\hline & Vane & Blade \\
\hline Hub radius [mm] & 32 & 32 -inlet \\
& & 30.2 -outlet \\
Height [mm] & 15.4 & 17.4 \\
Axial length [mm] & 19.8 & 15.5 \\
Chord [mm] & 26.76 & 19.08 \\
Nr of elements & 16 & 23 \\
Rated nominal power [KW] & 146.5 & \\
Isentropic efficiency & $83 \%$ & \\
Nominal speed [rpm] & 80,000 & \\
Nominal expansion ratio & 2.37 & \\
\hline
\end{tabular}

reference pressure is 0 atmosphere and the rotational speed is $80,000 \mathrm{rpm}$. Also at the outlet, it was imposed the mass flow, namely $0.685 \mathrm{~kg} / \mathrm{s}$ per machine. In Fig. 4, the channel considered for the numerical study is presented with the respective boundary conditions. The turbulence model used for this numerical study is the k-epsilon model coupled with a wall function.

To verify that a proper mesh was created and to ensure an accurate prediction of parameters, especially in the walls vicinity, the cell distribution near the walls is verifying in terms of $y+$ parameter. Figure 5 presents the distribution of $y+$ across the rotor blade. The $y+$ values are acceptable considering the turbulence model used and the fluid evolution through the stage. As the main objective of this study is to determine the influence of the tip clearance variation on the thermodynamic performances of the turbine and not the precise results for every turbine map, no further analysis of the numerical results precision was conducted.

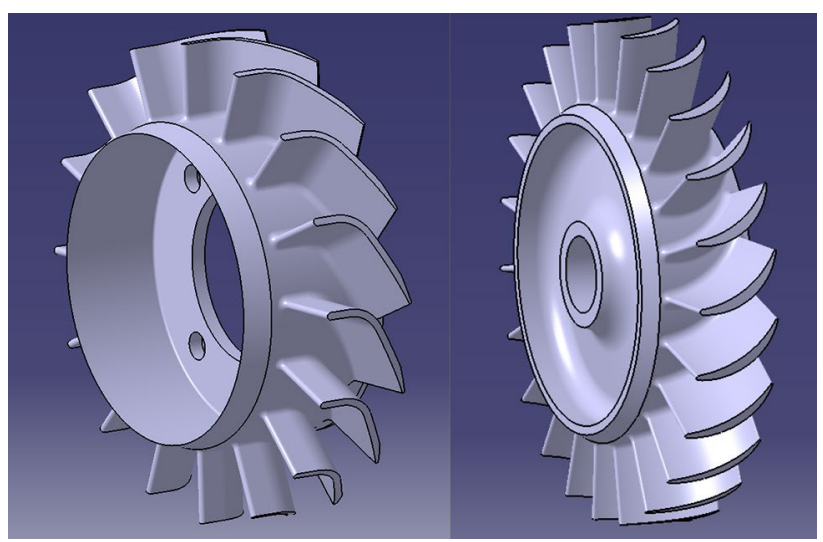

Fig. 2 3D models of turbine vane and rotor 


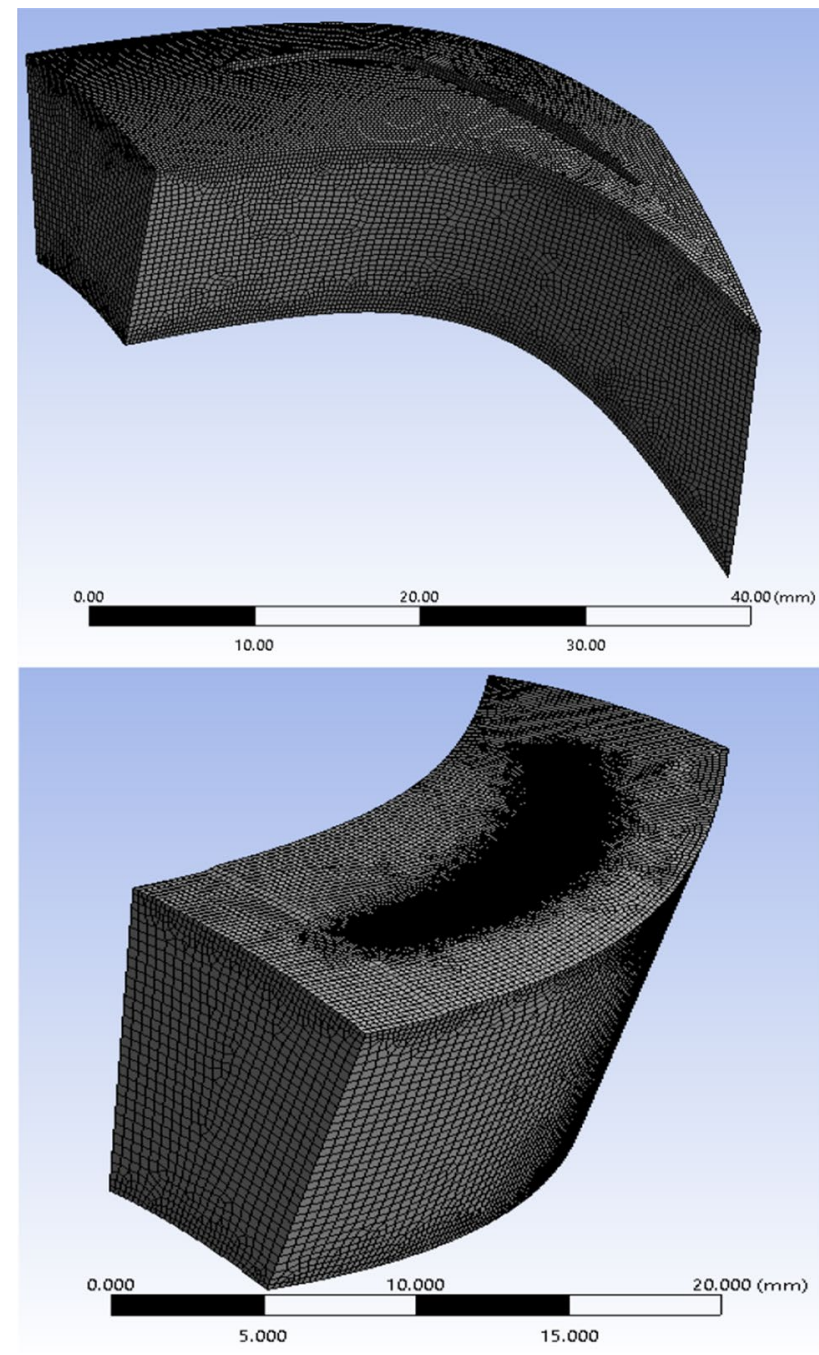

Fig. 3 Numerical grid for stator and rotor blade

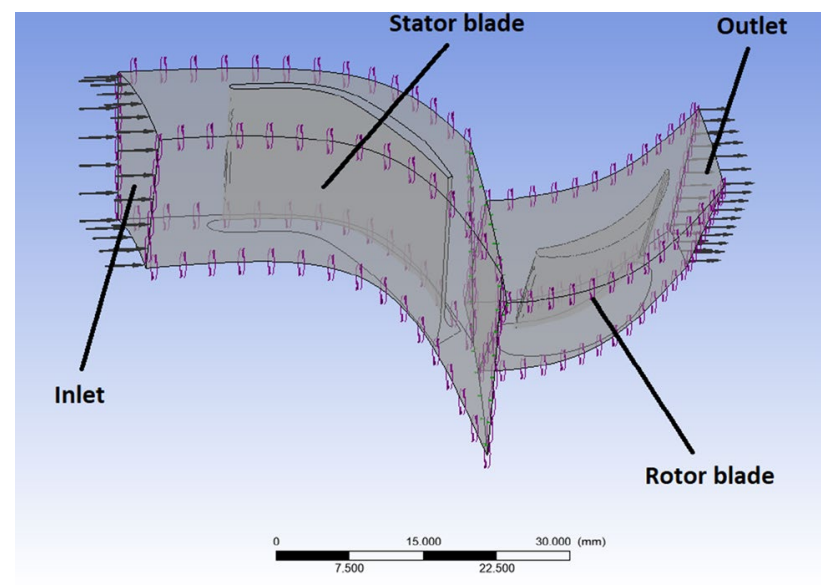

Fig. 4 The computational domain

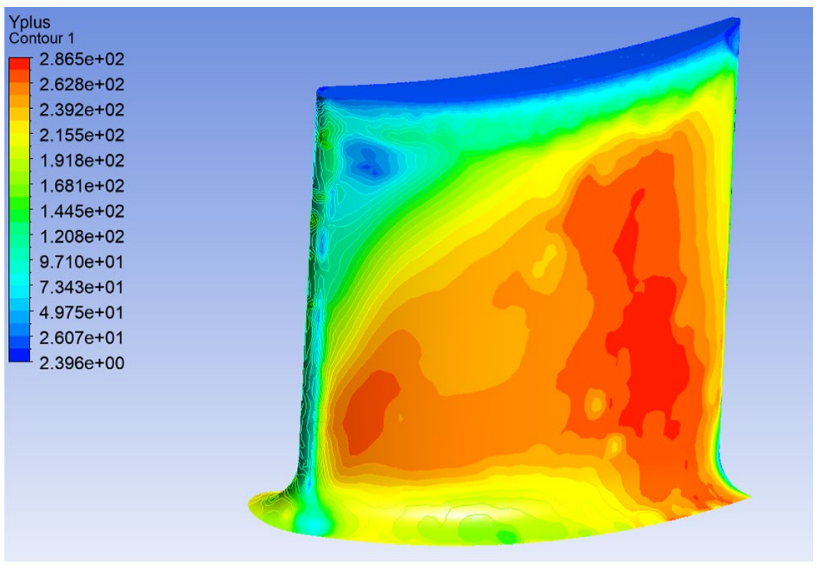

Fig. $5 y+$ distribution across rotor blade

\section{Results and Discussion}

For this study, five models were used, corresponding to five values of the clearance gap, from an ideal configuration, without a clearance gap to a large gap, corresponding to approximately $1.1 \%$ of blade chord. In each case, the blade geometry is identical, with the exception of the rotor shroud which increases with the value of the respective clearance gap. For each of the five computed cases, major changes in flow next to the tip of the blade and changes in the mass flow that passes through the gap were observed. Figure 6 presents the flow through a section near the rotor shroud and can be deduced, as expected, that the flow through this section and the influence on the flow in the vicinity is proportional with the clearance gap.

The turbine performances can be compared for different tip clearances by comparing the turbine characteristics. To obtain the complete maps, 20 cases were calculated for each clearance gap. By using specific parameters, calculated from the numerical results, the maps were drawn. The specific parameters are defined in Eq. (1) [4]:

$$
\left\{\begin{array}{l}
\Delta h_{\text {Tred }}^{*}=f\left(\dot{M}_{g} \frac{\sqrt{T_{3}^{*}}}{p_{3}^{*}}, \frac{n}{\sqrt{T_{3}^{*}}}\right), \\
\eta_{T}^{*}=f\left(\dot{M}_{g} \frac{\sqrt{T_{3}^{*}}}{p_{3}^{*}}, \frac{n}{\sqrt{T_{3}^{*}}}\right)
\end{array},\right.
$$

where: $\Delta h_{\text {Tred }}^{*}$-specific enthalpy drop; $\eta_{T}^{*}$-turbine efficiency; $\dot{M}_{g} \frac{\sqrt{T_{3}^{*}}}{p_{3}^{*}}$ reduced mass flow; $\frac{n}{\sqrt{T_{3}^{*}}}$ reduced rotational speed.

Using ANSYS CFX, four rotational speeds were simulated (80, 60, 40 and 20,000 rpm), for each of the five mass flows (representing 100\%, 95\%, 90\%, 80\%, respective $70 \%$ 
Fig. 6 The flow near the rotor blade tip for a $0.3 \mathrm{~mm}, \mathbf{b}$ $0.25 \mathrm{~mm}, \mathbf{c} 0.2 \mathrm{~mm}, \mathbf{d} 0.15 \mathrm{~mm}$, e $0 \mathrm{~mm}$ clearance gap
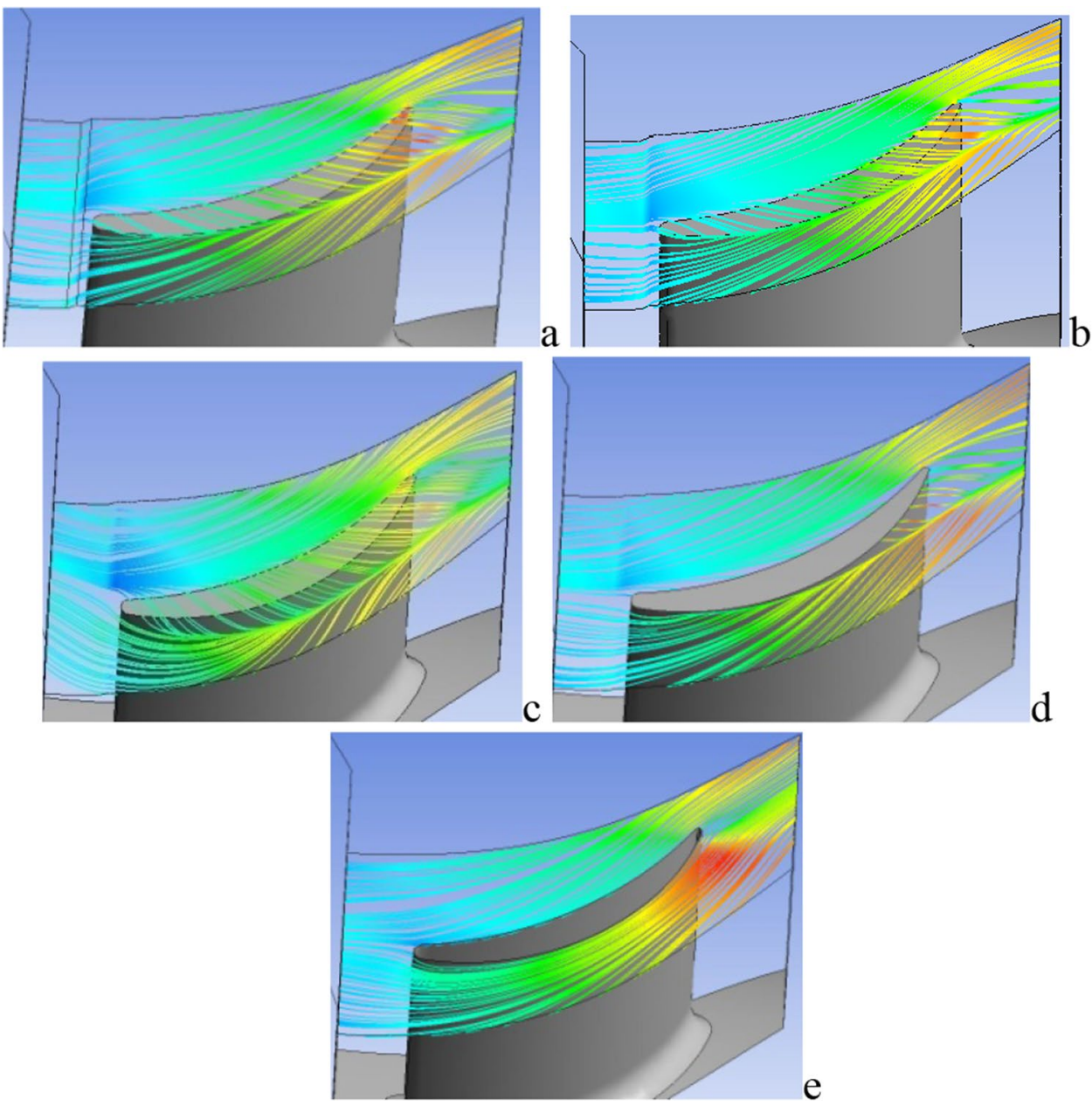

\section{$0.2 \mathrm{~mm}$ clearance}

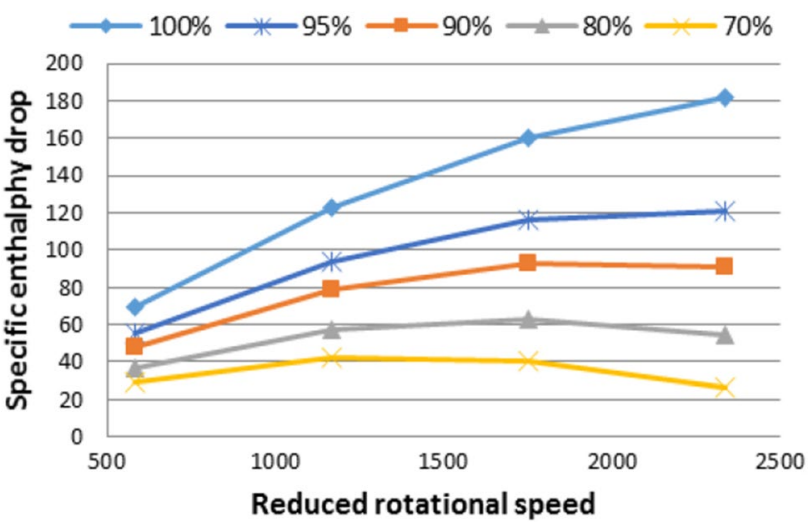

Fig. 7 Turbine map for $0.2 \mathrm{~mm}$ clearance gap

of nominal mass flow). In Fig. 7, it is shown the turbine map for $0.2 \mathrm{~mm}$ clearance gap.

The influence of the clearance gap in turbine performances can be seen in Fig. 8 for the higher regimes (100\%

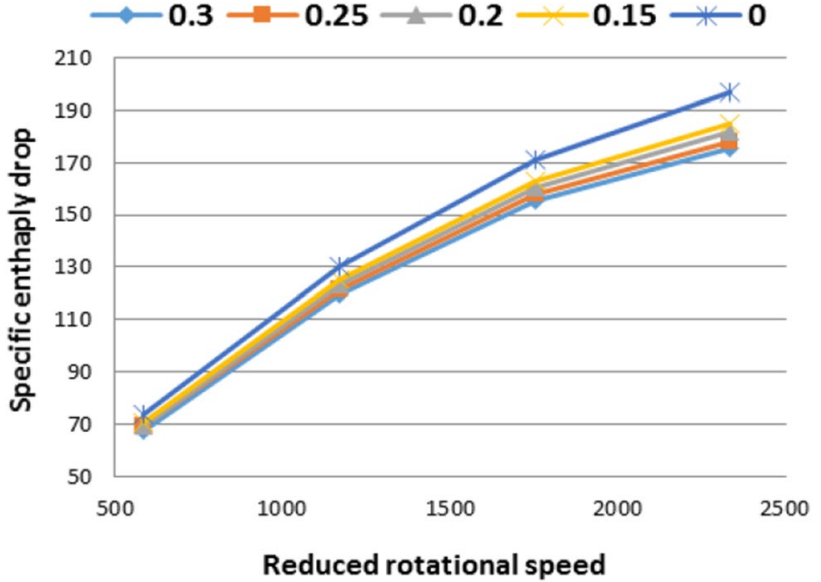

Fig. 8 Turbine performance comparison for five values of the clearance gap (100\% mass flow)

mass flow) and in Fig. 9 (70\% mass flow). By increasing the gap, the performances drop.

By comparing the effect of the clearance gap on the turbine performances, for higher and lower regimes, can be 


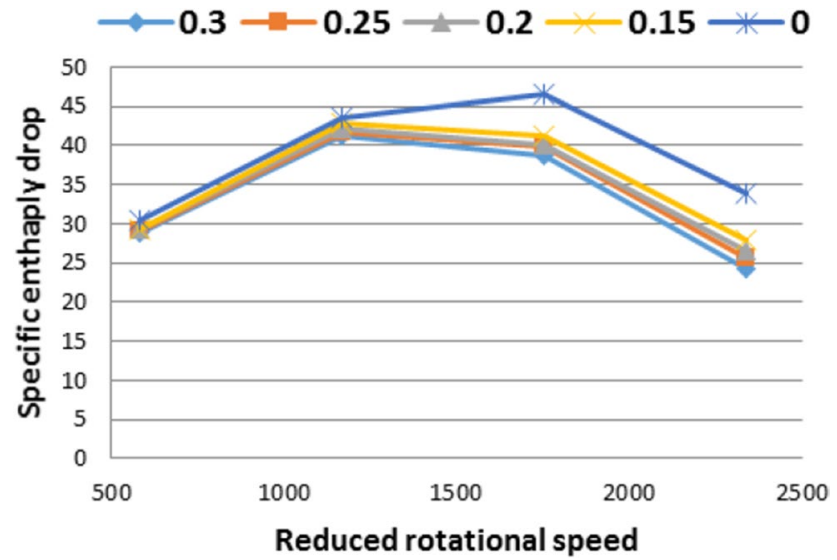

Fig. 9 Turbine performance comparison for five values of the clearance gap (70\% mass flow)

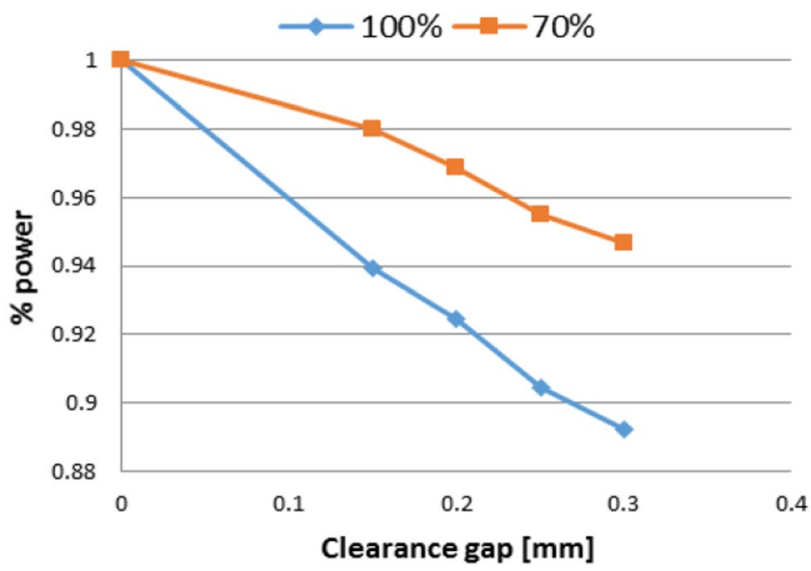

Fig. 10 Power variation with the clearance gap for higher and lower regimes

seen, from Fig. 10, that at higher regimes the clearance gap has a higher impact on the performance. A more than $10 \%$ drop in power was obtained for the maximum power regime and approximately $5.3 \%$ for a lower regime $(70 \%$ mass flow). In both cases, the curves presents a linear decrease in performance.

The maximum gap considered in this paper is $0.3 \mathrm{~mm}$ which represents approximatively $1.71 \%$ of blade height. In Table 2 there are present the percent of blade height and chord.

The present result agrees with the study of Haas and Kofskey [5] and Bindon [6] which determined a linear variation of performance with the clearance gap although they predicted a nonlinear relation for gaps less than $1 \%$ of chord.

To determine the influence of the clearance gap on the whole engine the compressor map is needed. Both the compressor and the turbine map are completed by using commercials programs like Smooth C respectively Smooth $\mathrm{T}$
Table 2 Gap percent of blade height and chord

\begin{tabular}{lll}
\hline Clearance gap & Blade height $(\%)$ & Chord $(\%)$ \\
\hline 0.3 & 1.71 & 1.12 \\
0.25 & 1.43 & 0.93 \\
0.2 & 1.14 & 0.75 \\
0.15 & 0.86 & 0.56 \\
0 & 0 & 0 \\
\hline
\end{tabular}

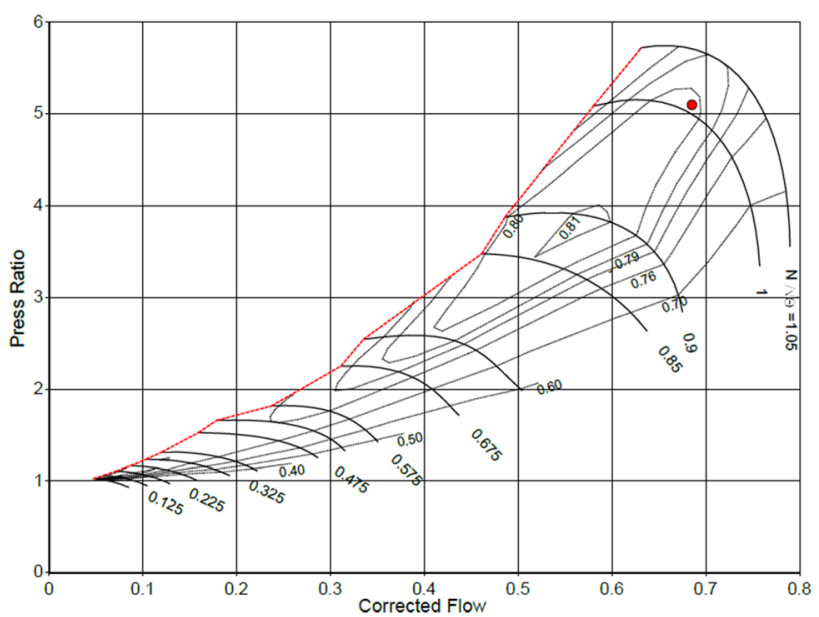

Fig. 11 Compressor map

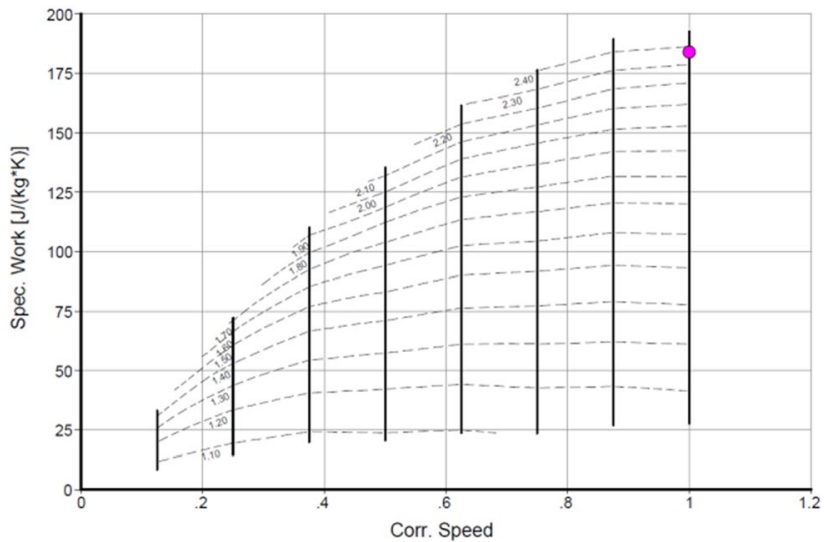

Fig. 12 Turbine map

and GasTurb. The performance map of the single stage, 5.1 nominal pressure ratio, the radial compressor is presented in Fig. 11.

The turbine map for an ideal configuration without clearance gap is presented in Fig. 12.

Using Transient module in GasTurb 13, the engine acceleration process from idle to maximum power was simulated by the use of engine cycle and component maps. For each clearance gap, a turbine performance characteristic was 


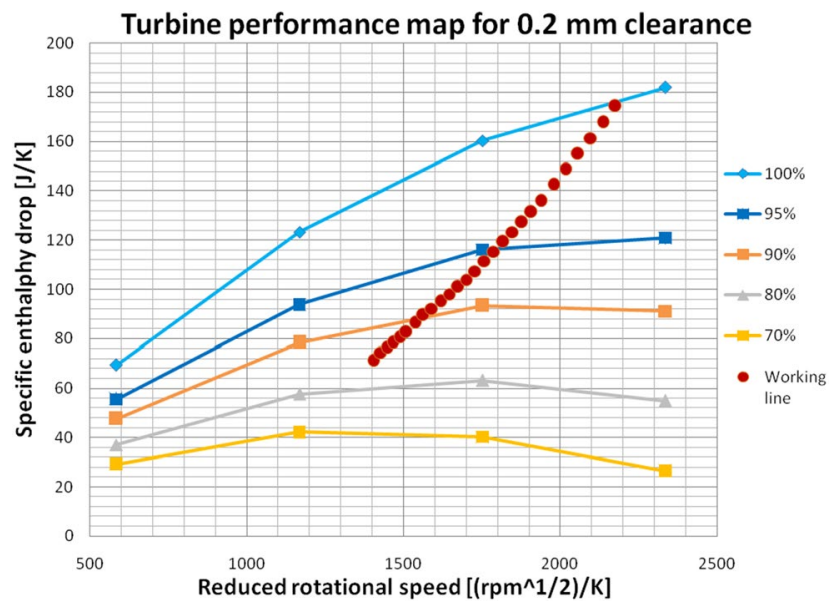

Fig. 13 Working line on the turbine performance map

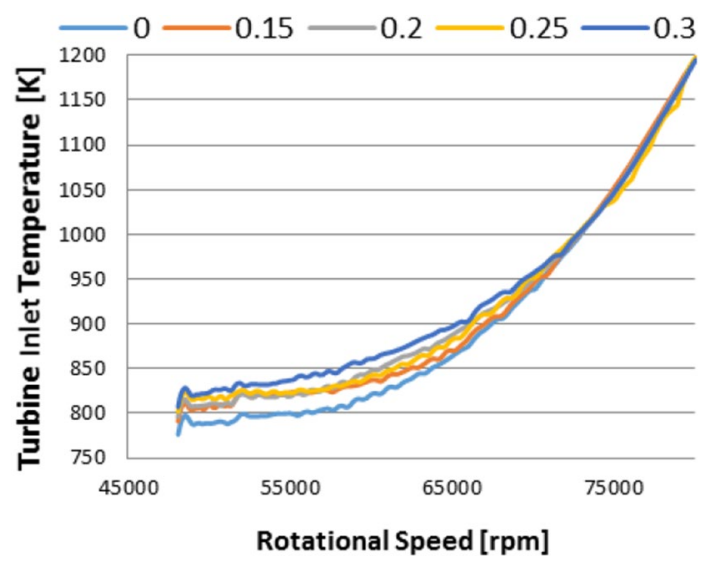

Fig. 14 Turbine inlet temperature variation during dynamic operation

created and then the acceleration process was simulated. Figure 13 presents the working line on the turbine performance map (determined for a constant $0.2 \mathrm{~mm}$ clearance gap) for the process considered.

In Fig. 14, it is presented the turbine inlet temperature evolution for each case during the dynamic process.

Because of the drop in turbine performances, with the increase of the clearance gap, to supply the necessary power to the compressor, a higher turbine inlet temperature is needed. Thus, we cannot correctly approximate the working line with only one value of the clearance gap.

By approximating the dynamic of the clearance gap and by using the working lines calculated before we can draw a more precise working line. The variation of clearance gap was approximated by scaling a typical evolution for axial turbines (presented in Fig. 1). On the rotational speed inter$\mathrm{val}$, from idle to nominal speed $(45,000-80,000 \mathrm{rpm})$, the tip clearance variation was determined to achieve the designed
Table 3 Turbine maps variation during acceleration

\begin{tabular}{lll}
\hline Rotational speed interval & $\begin{array}{l}\text { Reduced rotational } \\
\text { speed interval }\end{array}$ & Clearance gap \\
\hline $48,000-50,000$ & $1400-1460$ & 0.3 \\
$50,000-52,000$ & $1460-1518$ & 0.25 \\
$52,000-54,000$ & $1518-1576$ & 0.2 \\
$54,000-57,000$ & $1576-1666$ & 0 \\
$57,000-66,000$ & $1666-1929$ & 0 \\
$66,000-74,000$ & $1929-2166$ & 0.15 \\
$74,000-80,000$ & $2166-2323$ & 0.2 \\
\hline
\end{tabular}

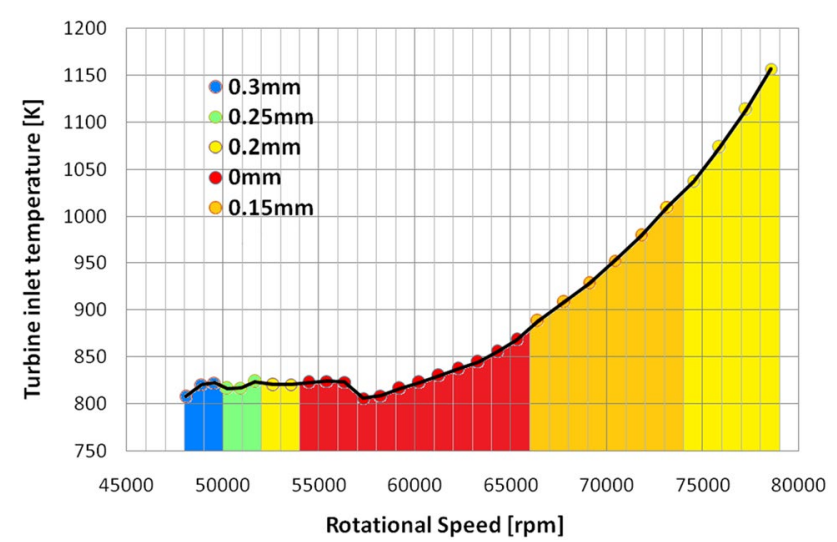

Fig. 15 Turbine inlet temperature variation considering multiple clearance gaps

gap $(0.2 \mathrm{~mm})$ at a nominal point. Thus, the turbine maps have been used as presented in Table 3 .

Figure 15 presents the turbine inlet temperature variation during acceleration corrected with the dynamic variation of the clearance gap.

A similar curve describes the fuel flow variation in Fig. 16. Knowing the precise power needed by the compressor, the correct temperature can be determined, thus the fuel flow needed can be predicted. For this simulation, the fuel flow used for the acceleration process, considering also the variation of the clearance gap using multiple turbine maps, was approximately $1.5 \%$ lower (globally) than considering only the nominal clearance gap. This value of lower fuel flow needed was calculated across the entire acceleration process, the clearance gap being lower or greater than the nominal one at respective regimes, thus the savings in fuel is limited. As for the maximum difference in the fuel flow, in this simulated process, an approximately $8 \%$ higher value was used which would determine a raise in temperature by 20 degrees, compared with the optimal acceleration process.

The working line from Fig. 17 was calculated across multiple turbine maps as it is described in Table 2. 


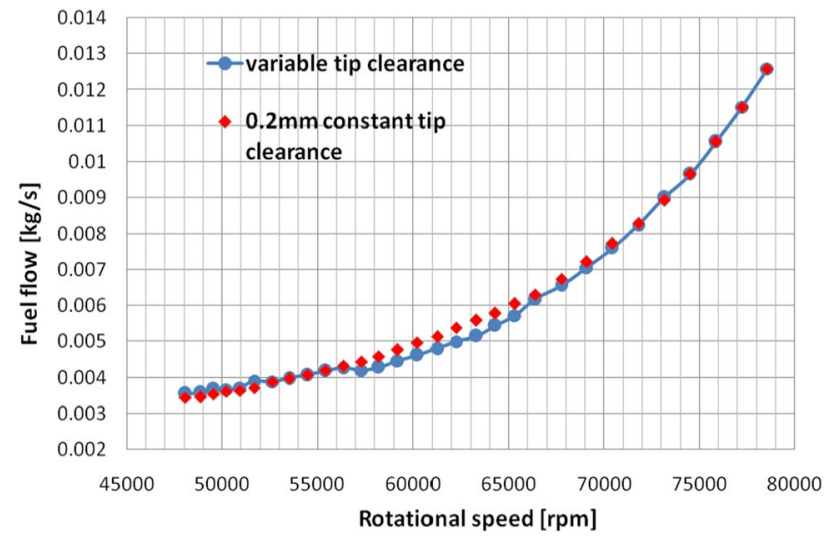

Fig. 16 Fuel flow consumed considering constant and variable clearance gaps

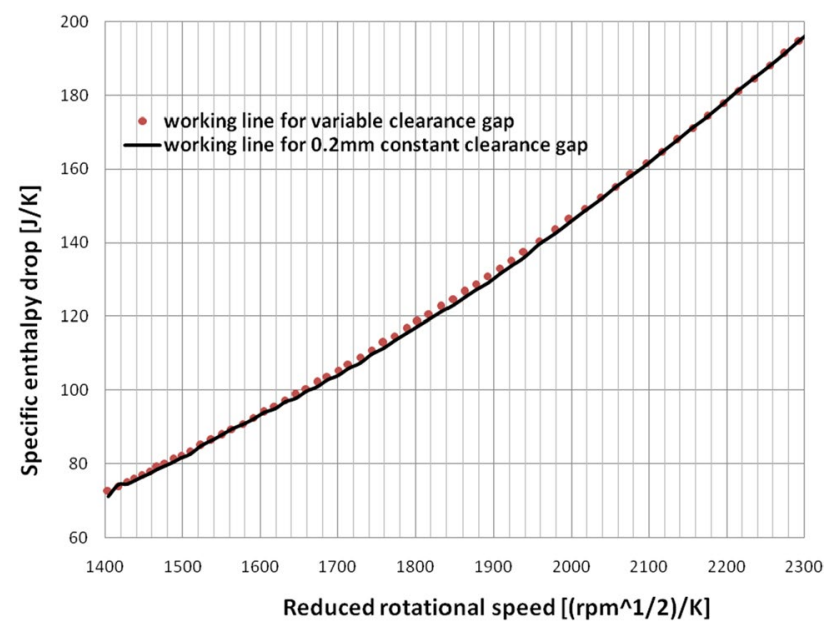

Fig. 17 Comparison between working lines for constant and variable clearance gap during simulating acceleration process

\section{Conclusions}

This paper conducted a study of the effect of the clearance gap on the turbine and engine performances during dynamic operations. Using a model of the dynamic response of the clearance gap, a few values were determined and then for each value a complete turbine map was created using CFD calculation and specialized commercial programs like Smooth C respectively Smooth T. By comparing the results, it was determined that the clearance gap effects on the turbine performances are more visible at higher regimes. The turbine inlet temperatures, required for acceleration in this simulation, considering multiple turbine maps, was determined as well as the fuel flow. It was determined that a $1.5 \%$ lower fuel flow was needed by considering the variation of the clearance gap during acceleration. The working line was also described in this paper across multiple turbine maps.

Future work should concentrate on determining an empirical coefficient to correct the fuel flow calculated to consider the effect of the clearance gap variation on the turbine performance. In addition, an experimental validation on these results is required.

Acknowledgements This work was carried out within POCA1-A1.2.3-G-2015, ID/SMIS code: P_40_422/105884, "TRANSCUMAT” Project, Grant No. 114/09.09.2016 (Subsidiary Contract No. 6/D.1.4/114/18.06.2018), Project supported by the Romanian Minister of Research and Innovation.

\section{Compliance with Ethical Standards}

Conflict of Interest The authors declares that there is no conflict of interest.

Open Access This article is licensed under a Creative Commons Attribution 4.0 International License, which permits use, sharing, adaptation, distribution and reproduction in any medium or format, as long as you give appropriate credit to the original author(s) and the source, provide a link to the Creative Commons licence, and indicate if changes were made. The images or other third party material in this article are included in the article's Creative Commons licence, unless indicated otherwise in a credit line to the material. If material is not included in the article's Creative Commons licence and your intended use is not permitted by statutory regulation or exceeds the permitted use, you will need to obtain permission directly from the copyright holder. To view a copy of this licence, visit http://creativecommons.org/licenses/by/4.0/.

\section{References}

1. Denton J (1993) Loss mechanisms in turbomachines. ASME J Turbomach 115(4):621-656

2. Japikse D, Baines NC (1994) Introduction to turbomachinery. Concepts ETI, Inc. and Oxford University Press, Vermont, pp 6-35

3. Moustapha H, Zelesky MF, Baines NC, Japikse D (2003) Axial and radial turbines. Concepts ETI, Inc. D.B.A. Concepts NREC, Vermont

4. Ainley DG, Mathieson GCR (1951) A method of performance estimation for axial-flow turbines. Aero Res Council Reports And Memoranda No. 2974

5. Haas EJ, Kofskey GM (1979) Effect of rotor tip clearance and configuration on overall performance of a 12.77-centimeter tip diameter axial-flow turbine. In: Proceedings of the ASME 1979 international gas turbine conference and exhibit and solar energy conference. Gas turbines, vol 1A, SanDiego, California, USA. 12-15 March 1979. V01AT01A042. ASME

6. Bindon JP (1989) The measurement and formation of tip clearance loss. J Turbomach 111:258-263

Publisher's Note Springer Nature remains neutral with regard to jurisdictional claims in published maps and institutional affiliations. 